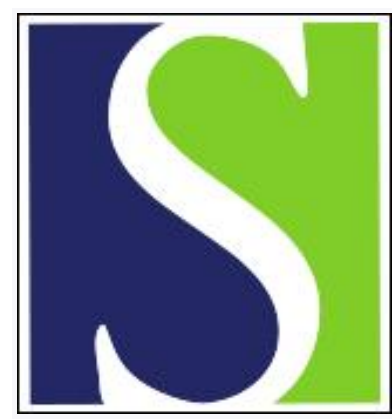

Scand J Work Environ Health 2009;35(3):180-187

https://doi.org/10.5271/sjweh.1324

Published online: 08 May 2009, Issue date: 00 May 2009

Sleep and autonomic nervous system changes - enhanced cardiac sympathetic modulations during sleep in permanent night shift nurses

by Chung M-H, Kuo TBJ, Hsu N, Chu H, Chou K-R, Yang CCH

Affiliation: Graduate Institute of Nursing, Taipei Medical University, Taipei, Taiwan.

The following articles refer to this text: 2010;36(2):81-84;

2010;36(6):515-516; 2012;38(2):171-181

Key terms: autonomic nervous system; daytime sleep; EEG; electroencephalographic delta-power; electroencephalography; enhanced cardiac sympathetic modulation; heart rate variability; night shift; night shift nurse; nighttime sleep; nurse; permanent night shift nurse; shift work; sleep

This article in PubMed: www.ncbi.nlm.nih.gov/pubmed/19430710 


\title{
Sleep and autonomic nervous system changes - enhanced cardiac sympathetic modulations during sleep in permanent night shift nurses
}

\author{
by Min-Huey Chung, PhD, ${ }^{1,2}$ Terry BJ Kuo, PhD, ${ }^{3,4}$ Nanly Hsu, PhD, ${ }^{5} \mathrm{Hsin}$ Chu, PhD, ${ }^{6}$ Kuei-Ru Chou, \\ PhD, ${ }^{1}$ Cheryl CH Yang, PhD ${ }^{3,4}$
}

\begin{abstract}
Chung MH, Kuo TBJ, Hsu N, Chu H, Chou KR, Yang CCH. Sleep and autonomic nervous system changes enhanced cardiac sympathetic modulations during sleep in permanent night shift nurses. Scand J Work Environ Health 2009;35(3):180-187.
\end{abstract}

\begin{abstract}
Objectives Disturbed sleep is the most common problem among the many health-related effects of shift work, with shift workers clearly having higher rates of cardiac disorders. However, the possible mechanism underlying the related health effects of shift work has yet to be examined. Consequently, this study aimed to explore the influence of long-term night shift work on the sleep patterns of nurses and their cardiac autonomic nervous system during sleep.
\end{abstract}

Methods Our sample comprised ten permanent night shift and ten regular morning shift nurses. Nurses
slept in their dormitory where they were allowed to sleep and wake spontaneously. All sleep parameters were
digitized using an ambulatory polysomnographic recorder. Using sleep patterns and heart rate variability, the
day- and nighttime sleep of permanent night shift nurses were compared with the nighttime sleep of regular
morning shift nurses.

Results Compared with the nighttime sleep of regular morning shift nurses, the pattern of daytime sleep of permanent night shift nurses showed significantly lower sleep onset latency. Permanent night shift nurses' daytime sleep also had greater proportions of Stage 3 and 4 (deep sleep), and arousal index than recorded during their nighttime sleep. Both the low frequency and low to high frequency ratio of the nighttime sleep of night shift nurses were significantly higher during periods of non-rapid eye movement (NREM) sleep than the nighttime sleep of morning shift workers. In addition, the electroencephalography delta-power of the nighttime sleep of night shift nurses was significantly lower during the first NREM episode sleep than those of both the daytime sleep of night shift workers and the nighttime sleep of morning shift nurses.

Conclusions Permanent night shift nurses have higher sympathetic activity during nighttime sleep than regular morning shift nurses. Night shift working may have effects on the sleeping patterns of nurses in the long run, inducing higher cardiac sympathetic regulation.

Key terms electroencephalographic delta-power; heart rate variability; nighttime sleep; daytime sleep; shift work.

After working all night, permanent night shift nurses must sleep during the day, in contradiction of their normal diurnal rhythm. As a result, clear indications of physiological desynchronization of circadian rhythms have been found amongst nighttime workers. This disturbance of biological rhythms can potentially result in sleep deprivation and act as a mediator of cardiovascular disease (1). Studies among papermill workers on night shift duty have shown that the quality of their daytime sleep is characterized by reduced total sleep and differs from nighttime sleep (2). For temporary night shift nurses, daytime sleep may be able to compensate for insufficient sleep. However, permanent night shift nurses may suffer from long-term sleep deprivation when sleeping during the day. This study aimed to examine whether sleep quality and sleep-related cardiac

1 Graduate Institute of Nursing, Taipei Medical University, Taipei, Taiwan.

2 Institute of Medical Sciences, Tzu Chi University, Hualien, Taiwan.

3 Institute of Brain Science, National Yang-Ming University, Taipei, Taiwan.

4 Sleep Research Center, National Yang-Ming University, Taipei, Taiwan.

5 Nursing Department, Yuanpei University, Hsinchu, Taiwan.

6 Institute of Aerospace Medicine, School of Medicine, National Defense Medical Center, Taipei, Taiwan.

Correspondence to: Dr CCH Yang, Institute of Brain Science, National Yang-Ming University, No 155, Section 2, Linong Street, Beitou District, Taipei 11221, Taiwan. [E-mail: cchyang@ym.edu.tw] 
autonomic function differ between nurses working night shifts and those working morning shifts.

Heart rate variability (HRV), a measure of the autonomic cardiac control, can be used as a marker of cardiac dysfunction (1). High frequency (HF) power mainly indicates cardiac vagal activity, whereas the low frequency (LF) to HF power ratio (LF : HF) reflects cardiac sympathetic modulations or sympathovagal balance (3). Reduced cardiac vagal tone or impaired vagal function has an increased risk of hypertension (4) and coronary heart disease (5). In addition, a significant reduction in the LF : HF power value has been found among nurses working night shifts compared to those who work in the morning or afternoon. This reduction in cardiac sympathetic modulation indicates that night shift nurses may well experience a reduction in alertness during their working hours (6).

There are, however, very few studies which have set out to examine sleep disorders amongst night shift nurses, as well as the resulting dysfunctions in the automatic nervous system which may be attributed to long-term night shift working. Various studies have found that chronic insomniacs have an increased risk of coronary artery disease and hypertension (7-9). Shift workers are prone to higher rates of cardiac, gastrointestinal and reproductive disorders $(10,11)$. Furthermore, shift working nurses have greater difficulty falling or staying asleep; they also frequently suffer from severe autonomic disturbances $(6,12)$. Thus, a relationship is discernible between shift working and autonomic nervous system disturbances among nurses.

Sleep is an important part of the life of humans and other mammals. However, several prior studies have demonstrated that the measurement of HRV while a person is awake may not reflect their HRV state during sleep $(13,14)$, since some delicate alternations - such as sympathetic activation accompanied by hypertension - are not readily detected without conducting a sleep study $(13,15)$. Recent improvements in sleep research techniques $(13,15-18)$ now allow us to test whether sleep patterns and sleep-related cardiac autonomic regulation are influenced by long-term night shift work in nurses. It therefore seems appropriate to undertake an exploration of the changes in HRV which accompany sleep, in order to clarify the pathways between cardiovascular disease and nurses engaged in shift working.

\section{Study population and methods}

\section{Participants and procedures}

The study participants were recruited from the MedicalSurgical Ward of the Songshan Armed Forces General Hospital. Circadian types were categorized as morning-, evening- and intermediate types (19). We used the Pittsburgh Sleep Quality Index (20) questionnaire to measure self-reported sleep habits over the previous one-month period; a higher score indicated poorer sleep quality.

No significant differences were found between permanent night shift nurses and regular morning shift nurses in terms of age, body mass index, years employed in nursing, blood pressure, tea or coffee consumption, sleep quality, and circadian type. All of the subjects who worked permanently on night shifts were currently unmarried; the choice of working at night was made mainly for financial reasons and because of the alternatives that such work offered. The study's exclusion criteria included psychopathology, neurological, or cardiovascular disease, or the use of any medication that influences sleep, the autonomic nervous system, or cardiovascular fluctuations (such as hypnotics and the subjects' self-reported caffeine or nicotine consumption). Informed written consent was obtained from all participants and the experimental protocol was approved by the Ethics Committee of Tri-Service General Hospital in Taiwan.

Nurses in the permanent night shift group worked three consecutive night shifts (23:30-07:30 hours). This was followed by two or three days off, and a further three or four consecutive nights of shift work. Nurses in the regular morning shift group worked five consecutive morning shifts (08:00-16:00 hours) per week, followed by two days off. After a day of habituation and following the second morning shift, polysomnographic recordings were performed during the nighttime sleep of regular morning shift nurses. Likewise, recordings were taken during the daytime sleep of the permanent night shift nurses after their second night shift. We indicated in a prior study that a period of at least two consecutive days off was sufficient for nurses working night shifts to return to the normal nighttime sleep patterns of dayshift workers (21). Therefore, a further recording of the nighttime sleep of permanent night shift nurses was carried out on their second day off. The experimental group, examining the nighttime and daytime sleep of permanent night shift nurses, was compared with the control group that looked at the nighttime sleep of regular morning shift nurses. All subjects were encouraged to continue to follow their usual sleeping habits.

\section{Data recording}

The recordings, which were carried out using electroencephalography (EEG, C3/Cz), electro-oculography (EOG), sub-mental electromyography (EMG) and electrocardiography (ECG), were synchronously digitized and stored in a memory card during the night or day using an ambulatory recorder. The filter bandwidths of 
the signals used different multipliers, similar to a previous study on data acquisition, as follows: EEG (5,000), EMG (2,000), ECG (5,000), and EOG (1,000) (22).

Both EEG and EMG were filtered with $0.32-40 \mathrm{~Hz}$, the EOG with $0.032-40 \mathrm{~Hz}$, and the ECG with 0.64 $40 \mathrm{~Hz}$. These bioelectric signals were relayed to an 8-bit analog-digital converter connected to an IBM PC-compatible computer. The EEG, EOG, EMG, and ECG signals were synchronously digitized but at different sampling rates $(128,128,128$, and $256 \mathrm{~Hz}$, respectively). The acquired data were analyzed online and stored simultaneously on a hard disk for subsequent offline verification.

\section{Digital signal processing}

For sleep stage analysis, the data file was converted into European Data Format and then imported into commercial sleep analysis software Somnologica 3.1.2 (Embla, Denver, CO, USA). The computer-assisted sleep analysis was carried out according to the criteria defined by Rechtschaffen \& Kales (24). The score arousal meant that the abrupt shift of EEG frequency (including alpha, theta, and/or frequencies greater than $16 \mathrm{~Hz}-$ but not spindles) lasted at least 3 seconds, with at least $10 \mathrm{sec}-$ onds of stable sleep preceding the change during sleep stages. The scoring of arousal required a concurrent increase in sub-mental EMG lasting at least 1 second during rapid eye movement (REM) sleep (23). However, the score of stage wakening had more than $50 \%$ of the epoch, either reactive alpha or age-appropriate dominant posterior rhythm, over the occipital region. Furthermore, the arousal index and awakening index indicated that both times of arousals and awakenings were divided into total sleep time (23). A qualified sleep technician verified the results. The pre-processing of the ECG signals was designed according to the recommended procedures (3) that have been described fully in previous reports (18, 24). In brief, the computer algorithm identified each normal ventricular discharge waveform and rejected each ventricular premature complex or noise according to its likelihood in a standard template. In order to assess the ventricular rate, stationary $\mathrm{R}-\mathrm{R}$ intervals were resampled and interpolated at a rate of $8 \mathrm{~Hz}$ to provide continuity in the time domain. The sampling rate of EEG signals was also reduced to $64 \mathrm{~Hz}$.

\section{Power spectral analysis}

In addition to the accompanying autonomic functions, we analyzed the sleep periods marked from the first to third non-rapid eye movement sleep (NREM1-3) and the first to third rapid eye movement sleep (REM1-3). The analyzed EEG and R-R signals were truncated into successive 64-second time segments (windows) with 50\% (32 seconds) overlapping. A Hamming window was applied to each time segment to attenuate the leakage effect (22). Our algorithm then estimated the power density of the spectral components based on the fast Fourier transformation.

The resulting power spectrum was corrected for attenuation resulting from sampling and the application of the Hamming window (24) and consequently underwent fast Fourier transformation. For each 64-second time segment, we quantified the R-R spectrogram's (16) power of the HF $(0.15-0.4 \mathrm{~Hz})$ and LF $(0.04-0.15 \mathrm{~Hz})$, in addition to the delta power $(0.5-4 \mathrm{~Hz})$ of the EEG spectrogram. The LF : HF power value was also calculated. The HF power value indicates cardiac vagal activity whereas LF : HF reflects cardiac sympathetic modulations or sympathovagal balance (3). EEG delta power is a characteristic of NREM and used to define deep (stages 3 and 4) sleep (23).

\section{Statistical analysis}

The first step of our analyses involved a comparison of the basic characteristics of the study subjects in order to ensure total comparability between the control and experimental groups. The demographic variables of employment, heart rate, blood pressure, tea/coffee consumption, type of morningness/eveningness, and the scores of sleep quality were presented as mean and standard deviation for continuous variables and numbers for categorical factors on which proper statistical had been tests performed.

The specific design included two scenarios: (i) comparisons between the nighttime and daytime sleep of permanent night shift nurses in the experimental group; and (ii) the comparison of both the nighttime and daytime sleep of permanent night shift nurses with a control group (ie, the nighttime sleep of regular morning shift nurses). The comparisons of the sleep pattern indices during REM or NREM periods of sleep were carried out using Mann-Whitney $U$ tests for regular morning shift nurses and permanent night shift nurses, and a Wilcoxon Sign-Rank test for the experimental group. A similar analysis strategy was then performed for the logarithmically transformed EEG delta-powers, HF, LF, and LF : HF power values of the R-R spectrogram in order to correct for the skewness of their distributions (24).

All of the statistical analyses in this study were undertaken using the SPSS 13.0 for Windows software package (SPSS Inc, Chicago, IL, USA), with a P-value of $<0.05$ considered as statistically significant.

\section{Results}

As shown in table 1, no significant differences were identified between the two groups according to the baseline comparisons; both groups comprised young 
and healthy nurses, and therefore provided a solid basis for comparison. The daytime sleep of permanent night shift workers was found to have significantly lower sleep onset latency, in comparison with the nighttime sleep patterns of the daytime workers. Nevertheless, as shown in table 2, the daytime sleep of permanent night shift workers was also found to have significantly greater proportions of stage 3 and 4 sleep, and arousal index, than the levels recording during their nighttime sleep. The EEG delta-power during NREM1 and arousal index of the nighttime sleep of permanent night shift nurses were significantly lower than those found both during their daytime sleep and the nighttime sleep of morning shift nurses (figure 1 and table 2).

As regards cardiac autonomic function, both the LF and LF : HF power values in the patterns of the nighttime sleep of permanent night shift nurses were found to be significantly higher than those of the nighttime sleep of morning shift nurses. Furthermore, as compared to the daytime sleep of permanent night shift

Table 1. The basic characteristics for comparisons of experimental and control groups. $(\mathrm{bpm}=$ beats per minute, $\mathrm{PSQI}=$ Pittsburgh Sleep Quality Index)

\begin{tabular}{|c|c|c|c|c|c|}
\hline \multirow[t]{2}{*}{ Variable } & \multicolumn{2}{|c|}{$\begin{array}{l}\text { Control group a } \\
\quad(N=10)\end{array}$} & \multicolumn{2}{|c|}{$\begin{array}{c}\text { Experimental } \\
\text { group }^{b}(\mathrm{~N}=10)\end{array}$} & \multirow[t]{2}{*}{ P-value } \\
\hline & Mean & SD & Mean & SD & \\
\hline Age (year) & 29.00 & 2.91 & 26.90 & 2.60 & 0.11 \\
\hline Years employed & 3.10 & 2.03 & 4.30 & 1.70 & 0.17 \\
\hline Body mass index & 20.00 & 1.14 & 20.34 & 1.83 & 0.62 \\
\hline Heart rate (bpm) & 76.70 & 7.89 & 77.40 & 4.55 & 0.81 \\
\hline $\begin{array}{l}\text { Diastolic blood } \\
\text { pressure }(\mathrm{mmHg})\end{array}$ & 71.40 & 5.17 & 71.30 & 7.51 & 0.97 \\
\hline $\begin{array}{l}\text { Systolic blood } \\
\text { pressure }(\mathrm{mmHg})\end{array}$ & 117.6 & 6.38 & 111.60 & 5.13 & 0.33 \\
\hline Global PSQI & 8.80 & 2.86 & 7.10 & 2.33 & 0.32 \\
\hline \multicolumn{6}{|c|}{ Tea consumption (number of nurses) } \\
\hline Never & & & 2 & & .. \\
\hline$<1$ per week & & & 1 & & 0.62 \\
\hline 1-2 per week & & & 2 & & .. \\
\hline 3-4 per week & & & 4 & & .. \\
\hline 5-7 per week & & & 1 & & .. \\
\hline \multicolumn{6}{|c|}{ Coffee consumption (number of nurses) } \\
\hline Never & & & 4 & & .. \\
\hline$<1$ per week & & & 2 & & 0.38 \\
\hline $1-2$ per week & & & 0 & &.. \\
\hline 3-4 per week & & & 0 & & .. \\
\hline 5-7 per week & & & 0 & & .. \\
\hline \multicolumn{6}{|c|}{ Diurnal type (number of nurses) } \\
\hline Morningness & & & 2 & & 0.23 \\
\hline Neither & & & 6 & & .. \\
\hline Eveningness & & & 2 & & .. \\
\hline
\end{tabular}

a Regular morning shift nurses.

${ }^{b}$ Permanent night shift nurses.

c Mann-Whitney U tests for continuous variables and Chi-square tests for categorical variables. nurses, their nighttime sleep patterns exhibited higher LF and LF : HF power values, albeit with no statistical significance (table 3 ).

The HRV analyses from the REM1-3 and NREM1-3, summarized in figure 2, reveal slight, but important, differences between the different shifts. The R-R of the nighttime sleep of the permanent night shift nurses during NREM2, REM2, and REM3 and the R-R of their daytime sleep during REM3 were found to be significantly higher than that of the nighttime sleep of regular morning shift nurses. The LF power value of the nighttime sleep of the permanent night shift nurses during NREM1-3, REM1, and REM2 sleep periods was significantly higher than that of the morning shift nurses, as was the LF power value of the daytime sleep of night shift nurses during NREM1-3, and REM1, and REM3 sleep periods. The LF : HF power value of the nighttime sleep of nightshift nurses during NREM1-3 and REM1 sleep periods, and that of their daytime sleep during NREM3 were also found to be significantly higher than that of morning shift nurses. Finally, within the experimental group, the LF : HF power value during the sleeping periods of the daytime sleep of permanent night shift nurses was found to be significantly lower than that of their nighttime sleep during NREM1 and REM1.

\section{Discussion}

This paper indicated that the sleep patterns and cardiac autonomic activity during the sleep of nurses working only night shifts differed from those working only morning shifts. We found that, compared to the latter, nurses working night shifts had significantly higher LF

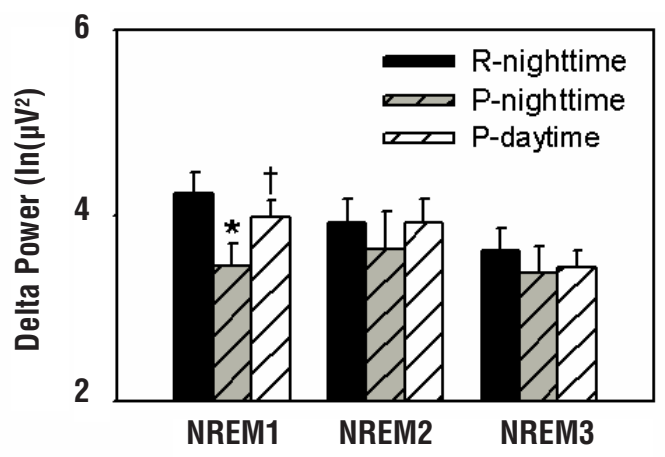

Figure 1. Electroencephalographic delta-power magnitude during 1-3 periods of non rapid eye movement (NREM) and rapid eye movement (REM). (R-nighttime = nighttime sleep of regular morning shift; P-nighttime $=$ the nighttime sleep of permanent night shift nurses; P-daytime $=$ the daytime sleep of permanent night shift nurses. Data are expressed as standard error of the mean; $N=10$ nurses per group) ${ }^{*} \mathrm{P}<0.05$ versus $\mathrm{R}$-nighttime.

$\dagger P<0.05$ versus $P$-nighttime. 
and cardiac LF : HF power values, particularly during periods of NREM sleep. Furthermore, compared to their nighttime sleep periods, the permanent night workers' daytime sleep showed significantly higher proportions of slow wave or deep sleep, arousal index, and EEG delta-power during NREM1 sleep. Based on these different sleep patterns, we found that cardiac LF was consistently higher amongst permanent night shift nurses independent of nighttime or daytime sleep.

The findings of this study suggested that nighttime workers would seem to have higher cardiac sympathetic regulations attributable to long-term night shift working, which may explain why they have been found to have higher rates of cardiac disorders. Sleep assigned according to a predetermined laboratory schedule can sometimes fail to reflect the habitual sleeping time (27-29). Our design allowed nurses to sleep spontaneously in their own beds, in privacy, and according to their flexible night shift sleeping schedule.

The spectral analysis of $\mathrm{R}-\mathrm{R}$ intervals assessing HRV reported a lower LF : HF value during periods of

Table 2. The differences of sleep patterns between shifts. (Rnighttime = the nighttime sleep of regular morning shift nurses, P-nighttime $=$ the nighttime sleep of permanent night shift nurses, P-daytime = the daytime sleep of permanent night shift nurses, $\mathrm{REM}=$ rapid eye movement)

\begin{tabular}{|c|c|c|c|c|c|c|}
\hline \multirow[t]{3}{*}{ Variable } & \multirow{2}{*}{\multicolumn{2}{|c|}{$\begin{array}{l}\text { Control group } \\
(\mathrm{N}=10) \\
\text { R-nighttime }\end{array}$}} & \multicolumn{4}{|c|}{$\begin{array}{l}\text { Experimental group } \\
\qquad(\mathrm{N}=10)\end{array}$} \\
\hline & & & \multicolumn{2}{|c|}{ P-nighttime } & \multicolumn{2}{|c|}{ P-daytime } \\
\hline & Mean & SD & Mean & SD & Mean & SD \\
\hline $\begin{array}{l}\text { Total sleep time } \\
\text { (minutes) }\end{array}$ & 359.89 & 43.39 & 362.55 & 97.53 & 330.15 & 136.84 \\
\hline $\begin{array}{l}\text { Wake after sleep } \\
\text { onset (minutes) }\end{array}$ & 31.48 & 28.62 & 25.74 & 12.82 & 21.32 & 17.48 \\
\hline $\begin{array}{l}\text { Sleep onset } \\
\text { latency (minutes) }\end{array}$ & 15.50 & 12.92 & 14.45 & 20.06 & 4.65 & $1.73^{\mathrm{a}}$ \\
\hline Sleep efficiency (\%) & 0.89 & 0.08 & 0.90 & 0.07 & 0.92 & 0.07 \\
\hline $\begin{array}{l}\text { Stage I } \\
\text { Stage } 2 \\
\text { Stage 3-4 } \\
\text { REM }\end{array}$ & $\begin{array}{r}9.35 \\
62.17 \\
4.81 \\
23.67\end{array}$ & $\begin{array}{l}3.77 \\
7.90 \\
7.17 \\
6.96\end{array}$ & $\begin{array}{r}8.39 \\
61.44 \\
1.54 \\
28.63\end{array}$ & $\begin{array}{l}4.18 \\
8.17 \\
2.24 \\
7.46\end{array}$ & $\begin{array}{r}7.40 \\
60.44 \\
7.39 \\
23.17\end{array}$ & $\begin{array}{r}5.11 \\
10.84 \\
5.92^{\mathrm{b}} \\
5.76\end{array}$ \\
\hline $\begin{array}{l}\text { REM latency from } \\
\text { sleep onset (minutes) }\end{array}$ & 97.60 & 64.52 & 61.60 & 21.68 & 63.35 & 26.50 \\
\hline $\begin{array}{l}\text { Number of sleep stage } \\
\text { transitions per hour }\end{array}$ & 15.78 & 4.84 & 14.54 & 3.71 & 16.12 & 5.49 \\
\hline $\begin{array}{l}\text { Awakening index } \\
\text { (times/hour) }\end{array}$ & 4.02 & 1.49 & 4.53 & 1.42 & 3.22 & 1.26 \\
\hline $\begin{array}{l}\text { Arousal index } \\
\text { (times/hour) }\end{array}$ & 3.91 & 1.61 & 2.69 & $0.65^{\mathrm{a}}$ & 3.71 & $1.24^{b}$ \\
\hline $\begin{array}{l}\text { Delta power } \\
\left(\ln \left(\mu \mathrm{V}^{2}\right)\right.\end{array}$ & 3.82 & 0.75 & 3.41 & 0.78 & 3.74 & 0.63 \\
\hline
\end{tabular}

a Pairs with statistical differences for shifts in experimental group comparing to control group by Mann-Whitney U test; $P<0.05$ versus R-nighttime.

${ }^{\mathrm{b}}$ Statistical significance within experimental group by Wilcoxon Sign-Rank test; $\mathrm{P}<0.05$ versus $\mathrm{P}$-nighttime.
NREM sleep than during REM sleep amongst permanent night shift nurses; however, this study indicated that there were similar elevations during both NREM and REM sleep. Sleep characterized by autonomic nervous activity was found to be in line with the findings of prior studies $(30,31)$, which revealed a sympathetic dominance in the autonomic system during REM sleep. However, this result contradicted the expectation that vagal influence would be predominant during NREM sleep.

The present study demonstrated that, compared to regular morning shift nurses, permanent night shift nurses had higher LF : HF and LF power values; especially the LF value was shown to be significantly higher, independent of nighttime or daytime sleep. It is now becoming increasingly recognized that LF levels are influenced by both sympathetic and vagal modulations (3). This elevation of the LF value may explain the higher sympathetic activity induced and, thus, may prove to be a good marker for night shift nurses.
A
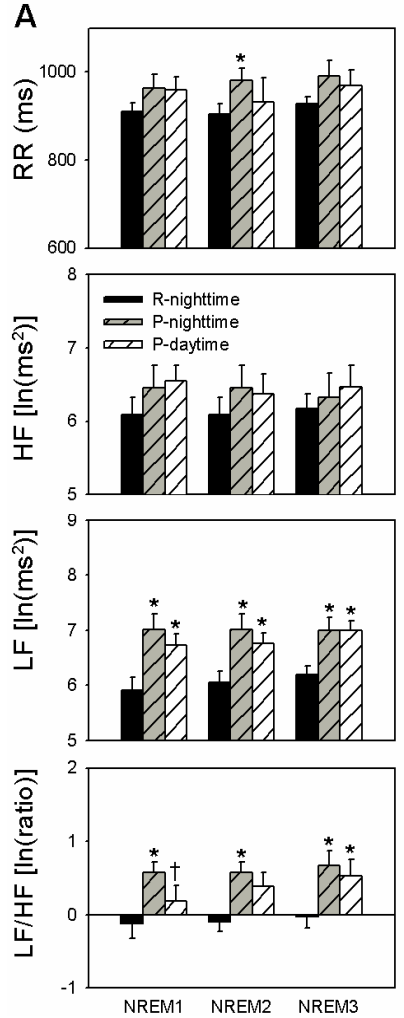

B
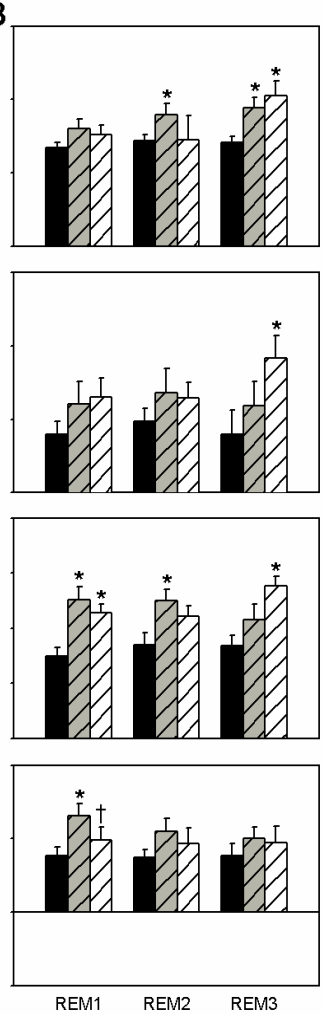

Figure 2. R-R intervals (RR), high-frequency power (HF), lowfrequency power (LF) and the LF to HF ratio (LF : HF) of heart rate variability among rotating three-shift nurses during 1-3 periods of non rapid eye movement (NREM) and rapid eye movement (REM) sleep. $(\mathrm{R}$-nighttime $=$ nighttime sleep of regular morning shift; $\mathrm{P}$ nighttime $=$ the nighttime sleep of permanent night shift nurses; P-daytime $=$ the daytime sleep of permanent night shift nurses. Data are expressed as standard error of the mean; $\mathrm{N}=10$ nurses per group) ${ }^{*} \mathrm{P}<0.05$ versus $\mathrm{R}$-nighttime.

$+\mathrm{P}<0.05$ versus $\mathrm{P}$-nighttime. 
A prior study indicated that a reduction in sympathetic activity, which could preserve the perfusion of the heart though an appropriate reduction in vascular resistance, may be responsible for attenuating the workload on the heart during periods of NREM sleep (32). In this study, the notable increase in sympathetic activity during periods of NREM sleep was found to lead to an increase in workload on the heart, which may explain the mechanisms linking shift work to cardiovascular events. We argue that there are several reasons why permanent night shift nurses have a higher HRV sympathetic activity during sleep.

Firstly, the shift schedule involved three or four night shifts in a row, followed by two days off between consecutive shift periods. When nurses are not working, they return to "normal" nighttime sleep schedules. That is, permanent night shift nurses usually sleep during the day, but sometimes sleep at night. Thus, night shift nurses become more biologically adapted to sleeping during the day and working throughout the night. However, no discernible differences were found during the waking hours of nurses working different shifts. This study also did not find any significant difference in terms of total sleep time and sleep efficiency to support this hypothesis of circadian adaptation (table 2).

Related studies have indicated that autonomic activity shows a diurnal pattern and that sympathetic activity

Table 3. Comparisons of cardiac autonomic functions between different shifts. (R-nighttime = the nighttime sleep of regular morning shift nurses; $P$-nighttime $=$ the nighttime sleep of permanent night shift nurses; $\mathrm{P}$-daytime = the daytime sleep of permanent night shift nurses; $R R=R-R$ intervals; $H F=$ high-frequency power; $L F=$ low-frequency power; LF : HF = LF to HF ratio)

\begin{tabular}{|c|c|c|c|c|c|c|}
\hline \multirow[t]{3}{*}{ Variable } & \multirow{2}{*}{\multicolumn{2}{|c|}{$\begin{array}{c}\text { Control group } \\
(\mathrm{N}=10)\end{array}$}} & \multicolumn{4}{|c|}{$\begin{array}{l}\text { Experimental group } \\
\qquad(\mathrm{N}=10)\end{array}$} \\
\hline & & & \multicolumn{2}{|c|}{ P-nighttime } & \multicolumn{2}{|c|}{ P-daytime } \\
\hline & Mean & SD & Mean & SD & Mean & SD \\
\hline \multicolumn{7}{|l|}{ Awake } \\
\hline $\begin{array}{l}\mathrm{RR}(\mathrm{ms}) \\
\mathrm{HF}\left[\ln \left(\mathrm{ms}^{2}\right)\right] \\
\mathrm{LF}\left[\ln \left(\mathrm{ms}^{2}\right)\right] \\
\mathrm{LF}: \mathrm{HF}(\text { ratio) }\end{array}$ & $\begin{array}{r}829.88 \\
6.05 \\
6.68 \\
0.66\end{array}$ & $\begin{array}{r}49.85 \\
0.71 \\
0.67 \\
0.45\end{array}$ & $\begin{array}{r}858.37 \\
6.30 \\
7.08 \\
0.79\end{array}$ & $\begin{array}{r}83.06 \\
0.84 \\
0.57 \\
0.44\end{array}$ & $\begin{array}{r}750.85 \\
6.38 \\
7.17 \\
0.79\end{array}$ & $\begin{array}{r}267.23 \\
0.79 \\
0.60 \\
0.75\end{array}$ \\
\hline \multicolumn{7}{|l|}{ NREM sleep } \\
\hline $\begin{array}{l}\mathrm{RR}(\mathrm{ms}) \\
\mathrm{HF}\left[\ln \left(\mathrm{ms}^{2}\right)\right] \\
\mathrm{LF}\left[\ln \left(\mathrm{ms}^{2}\right)\right] \\
\mathrm{LF}: \mathrm{HF}(\text { ratio })\end{array}$ & $\begin{array}{r}914.99 \\
6.22 \\
6.18 \\
-0.04\end{array}$ & $\begin{array}{r}52.42 \\
0.66 \\
0.58 \\
0.40\end{array}$ & $\begin{array}{r}990.40 \\
6.35 \\
7.01 \\
0.61\end{array}$ & $\begin{array}{c}86.39^{a} \\
0.90 \\
0.61^{a} \\
0.56^{a}\end{array}$ & $\begin{array}{r}963.95 \\
6.44 \\
6.84 \\
0.40\end{array}$ & $\begin{array}{r}97.26 \\
0.79 \\
0.47 \mathrm{a} \\
0.58\end{array}$ \\
\hline \multicolumn{7}{|l|}{ REM sleep } \\
\hline $\begin{array}{l}\mathrm{RR}(\mathrm{ms}) \\
\mathrm{HF}\left[\ln \left(\mathrm{ms}^{2}\right)\right] \\
\mathrm{LF}\left[\ln \left(\mathrm{ms}^{2}\right)\right] \\
\mathrm{LF}: \mathrm{HF}(\text { ratio })\end{array}$ & $\begin{array}{r}882.89 \\
5.91 \\
6.71 \\
0.79\end{array}$ & $\begin{array}{r}63.32 \\
0.56 \\
0.44 \\
0.35\end{array}$ & $\begin{array}{r}963.50 \\
6.30 \\
7.45 \\
1.14\end{array}$ & $\begin{array}{c}73.76^{\mathrm{a}} \\
0.92 \\
0.63^{\mathrm{a}} \\
0.48\end{array}$ & $\begin{array}{r}926.96 \\
6.34 \\
7.34 \\
1.01\end{array}$ & $\begin{array}{r}91.93 \\
0.74 \\
0.41 \\
0.50\end{array}$ \\
\hline
\end{tabular}

a Pairs with statistical differences for shifts in experiment group comparing to control group by Mann-Whitney $\mathrm{U}$ tests; $\mathrm{P}<0.05$ versus R-nighttime. is also prevalent during the working time (6). Thus, the sympathetic activity may be elevated during the nighttime, regardless of whether a subject is working or sleeping. Furthermore, the potential effects of the diurnal pattern on the autonomic activity were found to be related to higher sympathetic activity during periods of daytime sleep. This study indicated that the nighttime sleep of permanent night shift nurses showed significantly higher LF : HF values than that of the regular morning shift nurses, particularly during periods of NREM1 sleep. According to Endo et al, the rapid REM accumulation may be attributable to circadian influence, with the distribution of REM sleep being reliant upon the circadian phase over consecutive cycles (33). Therefore, we inferred that, as night shift nurses sometimes sleep during the day, the sleep pattern had a greater effect on the cardiac autonomic function during the first episode of REM sleep. However, the LF : HF value of the daytime and nighttime sleep of permanent night shift nurses was significantly higher during periods of NREM3 sleep, compared to the nighttime sleep of regular morning shift nurses. Parker et al reported that as there is normally a delay when a person gets out of bed, there will be a delay in the timing of the morning increase in myocardial ischemic episodes (34). Our results were in line with this previous study, which indicated that the augmentation of sympathetic activity was induced around 13:00 hours, proving an elevated risk of cardiovascular disease in the early afternoon.

Secondly, our study demonstrated that, compared to regular morning shift nurses, those working the night shift had lower EEG delta-power during both their nighttime and daytime sleep (table 2). However, the higher EEG delta activity during the daytime sleep of permanent night shift nurses was still found to be lower than that of regular morning shift nurses. EEG delta-power is a characteristic of NREM and is used to define deep sleep (23). Several prior experimental studies have revealed that slow wave activity during periods of NREM sleep depends on prior waking time $(33,35$, 36). As we found that the daytime sleep of permanent night shift nurses had lower sleep onset latency and significantly higher proportions of deeper sleep, we argue that they may have longer periods of restlessness and exhaustion before eventually falling asleep during the day, compared to at night. This also explains the obvious exhaustion of nurses following periods of nighttime working. Our results also indicated that the total duration of daytime sleep was a slightly shorter than that of nighttime sleep, which is consistent with several prior studies among healthy men $(2,33,35,37)$. In other words, there is a tendency for the sleep quality of permanent night shift nurses to deteriorate and for them to develop a small, but cumulative, sleep deficit. It has been shown that deeper sleep is accompanied by 
further suppression of the sympathetic function (16). Thus the higher sympathetic tone could be explained by the lower EEG delta-power and a higher proportion of deeper sleep.

Thirdly, frequent exposure to environmental factors (ie, light as well as social factors) may be another important issue. This is due to the fact that although permanent night shift nurses become accustomed to sleeping during the day, the influence of morning light is unavoidable before, during, and after periods of daytime sleep in the nurses' dormitory. Prior studies have indicated that exposure to bright light certainly enhances activity in the sympathetic nervous system (38). It is worth noting that the morning light (entering through the bedroom windows) appeared to have some effects on the autonomic nervous system of nurses. If this were the case, we could assume that, as time progresses, nurses would increasingly grow accustomed to their environment, resulting in subtle changes to their autonomic nervous system activity. Consequently, cumulative enhancement of sympathetic activity may be developed regardless of any modifications to social and/or domestic factors.

In the current study, none of the participants had children. Many of the nurses were able to minimize competing social factors to prioritize sleeping. Although the participants were encouraged to sleep as long as they could, we found that most permanent night shift nurses usually slept until the early afternoon, when they woke for lunch; they may then have elected to sleep for a while before commencing their night shift. The effects of this double period of sleep pattern on permanent night shift nurses are worthy of further investigation.

We identified changes in EEG, EMG, EOG, and HRV among permanent night shift nurses for reference purposes; however, consecutive monitoring of sleep over several nights is necessary to produce additional data to provide a more complete picture of the possible changes in sleeping patterns. The nurses who participated in the study had approximately two years of night shift work experience. In a future study, we plan to compare nurses with two years of night shift work experience with two groups of nurses - those with ten years of night shift work experience and those with less than one year of experience. These comparisons should help to provide a better understanding of how sleep might affect performance at work.

This study included only a small sample of nurses and had, as a result very low power, particularly in terms of finding differences between various shifts. However, our results clearly indicate that, in the long run, night working has effects on sleep-related cardiac autonomic function. This study provided only a brief picture of the daytime sleeping patterns of nurses who work three or four consecutive night shifts and then return to nighttime sleep during their days off. Sleeping patterns over several nights, as well as after long vacations, would warrant future study.

\section{Acknowledgements}

We would like to thank Ms HY Chuan for her excellent technical support. This study was supported by grant number 97A-C-P506 from the Taiwanese Ministry of Education's Aim for the Top University Plan and grant number NSC-97-2314-B-038-046-MY2 from National Science Council of Taiwan.

\section{References}

1. Knutsson A, Boggild H. Shiftwork and cardiovascular disease: review of disease mechanisms. Rev Environ Health. 2000;15:359-72.

2. Dahlgren K. Adjustment of circadian rhythms and EEG sleep functions to day and night sleep among permanent nightworkers and rotating shiftworkers. Psychophysiology. 1981;18:381-91.

3. Task Force of the European Society of Cardiology and the North American Society of Pacing and Electrophysiology. Heart rate variability: standards of measurement, physiological interpretation and clinical use. Circulation. 1996;93:1043-65.

4. Grossman P, Brinkman A, de Vries J. Cardiac autonomic mechanisms associated with borderline hypertension under varying behavioral demands: evidence for attenuated parasympathetic tone but not for enhanced beta-adrenergic activity. Psychophysiology. 1992;29:698-711.

5. Hayano J, Yamada A, Mukai S, Sakakibara Y, Yamada M, Ohte N, et al. Severity of coronary atherosclerosis correlates with the respiratory component of heart rate variability. Am Heart J. 1991;121:1070-9.

6. Furlan R, Guzzetti S, Crivellaro W, Dassi S, Tinelli M, Baselli G, et al. Continuous 24-hour assessment of the neural regulation of systemic arterial pressure and RR variabilities in ambulant subjects. Circulation. 1990;81:537-47.

7. Bonnet $\mathrm{MH}$, Arand DL. Heart rate variability: sleep stage, time of night, and arousal influences. Electroencephalogr Clin Neurophysiol. 1997;102:390-6.

8. Schwartz S, McDowell Anderson W, Cole SR, Cornoni-Huntley J, Hays JC, Blazer D. Insomnia and heart disease: a review of epidemiologic studies. J Psychosom Res. 1999;47:313-33.

9. Suka M, Yoshida K, Sugimori H. Persistent insomnia is a predictor of hypertension in Japanese male workers. J Occup Health. 2003;45:344-50.

10. Wilson JL. The impact of shift patterns on healthcare professionals. J Nurs Manag. 2002;10:211-9. 
11. Muecke S. Effects of rotating night shifts: literature review. J Adv Nurs. 2005;50:433-9.

12. Freitas J, Lago P, Puig J, Carvalho MJ, Costa O, de Freitas AF. Circadian heart rate variability rhythm in shift workers. J Electrocardiol. 1997;30:39-44.

13. Kuo TBJ, Lai CJ, Shaw FZ, Lai CW, Yang CCH. Sleep-related sympathovagal imbalance in SHR. Am J Physiol Heart Circ Physiol. 2004;286:H1170-6.

14. Baharav A, Kotagal S, Gibbons V, Rubin BK, Pratt G, Karin J, et al. Fluctuations in autonomic nervous activity during sleep displayed by power spectrum analysis of heart rate variability. Neurology. 1995;45:1183-7.

15. Chen HY, Kuo TBJ, Shaw FZ, Lai CJ, Yang CCH. Sleep-related vagotonic effect of zolpidem in rats. Psychopharmacology. 2005;181:270-9.

16. Yang CCH, Lai CW, Lai HY, Kuo TBJ. Relationship between electroencephalogram slow-wave magnitude and heart rate variability during sleep in humans. Neurosci Lett. 2002;329:213-6.

17. Kuo TBJ, Yang CCH. Scatterplot analysis of EEG slow-wave magnitude and heart rate variability: an integrative exploration of cerebral cortical and autonomic functions. Sleep. 2004;27:648-56.

18. Kuo TBJ, Shaw FZ, Lai CJ, Lai CW, Yang CCH. Changes in sleep patterns in spontaneously hypertensive rats. Sleep 2004;27:406-12.

19. Horne JA, Ostberg O. A self-assessment questionnaire to determine morningness-eveningness in human circadian rhythms. Int J Chronobiol 1976;4:97-110.

20. Buysse DJ, Reynolds CF, 3rd, Monk TH, Berman SR, Kupfer DJ. The Pittsburgh Sleep Quality Index: a new instrument for psychiatric practice and research. Psychiatry Res 1989;28:193-213.

21. Ahasan R, Lewko J, Campbell D, Salmoni A. Adaptation to night shifts and synchronisation processes of night workers. J Physiol Anthropol Appl Human Sci 2001;20:215-26.

22. Kuo TBJ, Chan SHH. Continuous, on-line, real-time spectral analysis of systemic arterial pressure signals. Am J Physiol 1993;264:H2208-13.

23. Rechtschaffen A, Kales A. A manual of standardized terminology, techniques, and scoring system for sleep stages of Human subjects. Psychopharmacology (Berl). 1968;181:270-9

24. Kuo TBJ, Lin T, Yang CCH, Li CL, Chen CF, Chou P. Effect of aging on gender differences in neural control of heart rate. Am J Physiol. 1999;277:H2233-9.
25. Luoh HF, Kuo TBJ, Chan SHH, Pan WH. Power spectral analysis of electroencephalographic desynchronization induced by cocaine in rats: correlation with microdialysis evaluation of dopaminergic neurotransmission at the medial prefrontal cortex. Synapse. 1994;16:29-35.

26. Cajochen C, Brunner DP, Krauchi K, Graw P, Wirz-Justice A. Power density in theta/alpha frequencies of the waking EEG progressively increases during sustained wakefulness. Sleep. 1995;18:890-4.

27. Finelli LA, Baumann H, Borbely AA, Achermann P. Dual electroencephalogram markers of human sleep homeostasis: correlation between theta activity in waking and slow-wave activity in sleep. Neuroscience. 2000;101:523-9.

28. Somers VK, Anderson EA, Mark AL. Sympathetic neural mechanisms in human hypertension. Curr Opin Nephrol Hypertens. 1993;2:96-105.

29. Ako M, Kawara T, Uchida S, Miyazaki S, Nishihara K, Mukai $\mathrm{J}$, et al. Correlation between electroencephalography and heart rate variability during sleep. Psychiatry Clin Neurosci. 2003;57:59-65.

30. Mancia G. Autonomic modulation of the cardiovascular system during sleep. N Engl J Med. 1993;328:347-9.

31. Endo S, Kobayashi T, Yamamoto T, Fukuda H, Sasaki M, Ohta T. Persistence of the circadian rhythm of REM sleep: a variety of experimental manipulations of the sleep-wake cycle. Sleep. 1981;4:319-28.

32. Parker JD, Testa MA, Jimenez AH, Tofler GH, Muller JE, Parker JO, et al. Morning increase in ambulatory ischemia in patients with stable coronary artery disease. Importance of physical activity and increased cardiac demand. Circulation. 1994;89:604-14.

33. Borbely AA, Baumann F, Brandeis D, Strauch I, Lehmann D. Sleep deprivation: effect on sleep stages and EEG power density in man. Electroencephalogr Clin Neurophysiol. 1981;51:483-95.

34. Dijk DJ, Brunner DP, Beersma DG, Borbely AA. Electroencephalogram power density and slow wave sleep as a function of prior waking and circadian phase. Sleep. 1990;13:430-40.

35. Åkerstedt T, Kecklund G, Knutsson A. Spectral analysis of sleep electroencephalography in rotating three-shift work. Scand J Work Environ Health. 1991;17(5):330-6.

36. Saito Y, Shimizu T, Takahashi Y, Mishima K, Takahashi K, Ogawa $Y$, et al. Effect of bright light exposure on muscle sympathetic nerve activity in human. Neurosci Lett. 1996;219:135-7.

Received for publication: 18 September 2007 